\title{
Are some C19orf12 variants monoallelic for neurological disorders?
}

Huma Tariq ${ }^{1,2}$, Jalil ur Rehman Butt ${ }^{3}$, Henry Houlden $^{1 *}$, Sadaf $_{\mathrm{Naz}^{2 *}}$

${ }^{1}$ Department of Neurogenetics, UCL institute of Neurology, Queen Square House, University College

London, London

${ }^{2}$ School of Biological Sciences, University of the Punjab, Lahore, Pakistan

${ }^{3}$ Punjab Institute of Neurosciences, Lahore General Hospital, Lahore, Pakistan

*Equal contribution

\section{Correspondence:}

Dr. Sadaf Naz, School of Biological Sciences, University of the Punjab, Quaid-i-Azam Campus, Lahore, 54590, Pakistan, naz.sbs@pu.edu.pk

Dr. Henry Houlden, Department of Neurogenetics, UCL institute of Neurology, Queen Square House, University College London, London, England, h.houlden@ucl.ac.uk

\section{Acknowledgements}

We thank the family members for participation in the study. We are grateful to Dr Rashid Imran and Dr. Thomas Bourinaris for help in clinical investigations of patients.

\section{Disclosure}

The authors declare no conflict of interest 
Neurodegeneration with brain iron accumulation (NBIA) are a group of clinically heterogeneous neurodegenerative disorders associated with mutations in different genes, which include PANK2, PLA2G6, CP, FTL, C19orf12, WDR45, COASY, and FA2H [1, 2]. Variants of C19orf12 result in recessively inherited mitochondrial protein associated neurodegeneration (MPAN), a typical but often clinically aggressive NBIA [1]. Knockdown of C19orf12 orthologues CG3740 and CG11671 in D. melanogaster induces neurodegeneration specific phenotype characterized by damage of brain tissue and abnormal locomotor function [3]. C19orf12 encodes a mitochondrial protein which has four isoforms, the largest of which consists of 152 amino acids (uniprot Q9NSK7-1). Most of the pathogenic variants have been identified in the last exon 3 of C19orf12 [1].

To date, 38 nonsense, frameshift, missense and splicing variants (http://www.hgmd.cf.ac.uk/ac/gene.php?gene=C19orf12, accessed April 2019) located in exons 2 and 3 of C19orf12 have been reported to cause MPAN [1, 4]. The missense variants are hypothesized to disturb the sub-cellular localization of the protein, whereas other variants have been predicted to result in loss of protein or produce truncated proteins with or without wrongly incorporated amino acids. Over the years, seven monoallelic variants of $C 19 \operatorname{orf} 12$ were reported to cause disease in seven heterozygous individuals with MPAN. Six of these patients had frameshift variants in exon 3, whereas the seventh was a carrier of a missense variant in the same exon. It was hypothesized that frameshift variants may confer gain-of-function effect and play a role in pathogenesis of disorder in heterozygous individuals [1, $5]$.

We recruited a family RDHT08 with three affected individuals. The proband, II-5 (Fig. 1A) was born after an uneventful pregnancy and achieved his early milestones adequately. Disease onset was at the 
age of eight years, manifested with frequent falls. It progressed to tip toe walking, hammer pes cavus toes, dystonic hands and feet, rigidity, speech problem and decreased visual acuity at the age of sixteen. The affected member II-4 was an 18 years old sister of the proband. She was also affected at eight years of age. Apart from the phenotypes described above, she experienced mild ataxia, sensory neuropathy and cognitive impairment. The younger 15 years old sister II-6, had the same age of onset and symptoms, but dystonia and difficulty in walking were more severe. Brain magnetic resonance imaging (MRI) for the proband revealed moderate periventricular white matter abnormalities (Fig. 1B) and mild hypointesities in the globus pallidus (Fig. 1C).

The DNA of two affected siblings II-4 and II-5 was subjected to whole exome sequencing. The candidate variants were prioritized and segregation analysis was completed. A homozygous variant c.199delG;p.(Ala67LeufsTer5) in C19orf12 (NM_001031726.3) was found to segregate with the phenotype (Fig.1D) in the three affected individuals of the family, and was heterozygous in six unaffected carriers.

This variant was previously identified as one allele in a French NBIA patient with another missense variant c.416 A>G;p.(Tyr139Cys) in compound heterozygous form, and in homozygous form in two affected MPAN patients [5, 6], but no expression analyses were performed. However, our experiments demonstrate that the level of mutant C19orf12 mRNA is almost negligible in affected individuals with c.416 A>G;p.(Tyr139Cys) homozygous variant (Fig.1E), which indicates that the mutant allele will not give rise to a truncated protein. Our results confirm that C19orf12 haploinsufficiency cannot be a mechanism of disease since all six individuals who are carriers of c.199delG;p.(Ala67LeufsTer5) variant are unaffected (Fig. 1A). These six individuals together with all other reported cases of more than 
seventy heterozygous unaffected obligate carriers and their unaffected heterozygous children emphasize the fact that presence of half of the normal levels of functional protein is enough to account for the wildtype phenotype in these individuals. The high number of heterozygous carriers who are completely unaffected, excludes the possibility of reduced penetrance of the variants.

A dominant-negative effect or gain-of-function effect can be considered to be a pathomechanism for one of the reported monoallelic variants of C19orf12, c.358dupG; p.A120G fsX32, where the size of the predicted mutant protein is expected to be the same as that of the wild-type protein [6]. This variant was reported to cause young onset classic MPAN in one patient and a relatively different phenotype of Parkinsonism and dementia in his deceased father [6]. A dominant-negative effect of this c.358dupG; p.A120G fsX32 variant can be only inferred till functional assays are performed. Another reported monoallelic variant which could cause disorder by a dominant-negative effect is p.(Gly69Arg). However, multiple heterozygous carriers of the same variant have no phenotype [5].

Additional support for recessive nature of C19orf12 variants also exist. The bi-allelic pathogenic variants c.191insG;p.(Val64GlyfsTer18), c.199delG;p.(Ala67LeufsTer5) c.404insT;p.(Met135IlefsTer15) and c.436-437insG;p.(Ala146GlyfsTer6) are predicted to add unique amino acids to the protein before truncation (Fig.1F), but do not cause symptoms in heterozygous carriers. Furthermore, the unaffected heterozygous individuals with the variants c.404insT;p.(Met135IlefsTer15) and c.436-437insG;p.(Ala146GlyfsTer6), are expected to have aberrant proteins of 150 or 152 amino acids, respectively, a length approximately equal to or identical to that of the wild-type protein $[5,7]$. This negates the hypothesis that aberrant C19orf12 protein could act in dominant-negative manner. 
Detailed review of the reports where monoallelic variants in heterozygotes were suggested to be responsible for disease phenotype shows the limitations in methodology of the studies. For example, the C19orf12 heterozygous variant c.244 A>T; p.(Lys82Ter) was present in an NBIA patient and in her unaffected mother as well. The authors of the study suggested the presence of a second unidentified variant which could account for the disorder in the patient [5]. There is a high chance that in some studies, the second pathogenic allele in the same gene was missed, perhaps due to it being located in the regulatory region or due to large deletions within the second allele. Digenic inheritance could be another, although a remote possibility for the disorder in these individuals affected with MPAN and having monoallelic C19orf12 variants. Follow up of patients with monoallelic C19orf12 variants may reveal a second pathogenic variant in the same or a different gene.

\section{Legends}

\section{Figure 1: Pedigree, MRI and sequence traces for family RDHT08}

1A: Pedigree of family RDHT08. The genotypes for C19orf12 variant c.199delG; p.(Ala67LeufsTer5) are indicated below the individual symbols of all participants.

1B: The axial section of brain showing moderate periventricular white matter abnormalities from MRI of the brain for the proband.

1C: The MRI of the brain of the proband shows the coronal section of brain with hypointensities in the globus pallidus (encircled).

1D: Partial sequence chromatograms indicating the wild type allele in a normal control, heterozygous family member and homozygous deletion mutation c.199delG;p.(Ala67LeufsTer5) in an affected member with C19orf12 variant. The position of the mutation is indicated by an arrow. (Colored figure can be viewed online) 
1E: C19orf12 gene expression in family RDHT08. Lanes 1 and 2 show the results for nested RT-PCR on cDNA library using gene specific primers. A 201bp (arrow on left side) product was obtained from cDNA of two carriers of the variant, whereas lane 3 shows extremely reduced or no amplification of required product from cDNA library of homozygous affected member. Lane 4, size standard. Lanes 5-7 show the amplification of GAPDH specific 496bp products from the same cDNA samples (indicated with an arrow on the right side) as a positive control.

1F: All monoallelic and some reported bi-allelic mutations in C19orf12. The variants shown above were reported as monoallelic. Variants shown below the gene structure are those which have been reported to cause disorder when they are bi-allelic. Frameshift variants are shown in red colour, whereas missense and nonsense variants are in black. The variant shown in blue has been reported to cause disease both as monoallelic as well as in bi-allelic condition. Variant reported in this study is indicated with an asterisk. (References for all variants: PMID 23269600, 22704260, 25962551, 29295770, 22508347, 23166001, 21981780). E1, E2, E3 represent exons 1 to 3, respectively of C19orf12. Slanting lines depict introns, and the horizontal black lines upstream and downstream the coding regions depict the 5' and 3' untranslated regions, respectively. (Colored figure can be viewed online).

\section{Funding}

HT was supported by HEC indigenous fellowship and an HEC IRSIP scholarship. This study was funded by research grant \#2877 by the Higher Education Commission, Islamabad, Pakistan to SN and by grant of UCL institute of Neurology from the Wellcome Trust.

\section{Compliance with ethical standards}

\section{Conflict of interest}


The authors declare no conflict of interest

\section{Ethical approval}

All procedures in study were in accordance to ethical guidelines approved by ethical committee of School of Biological Sciences, University of the Punjab.

\section{Informed consent}

Written informed consent was taken from all participants of the study.

\section{Disclosure concerning the present manuscript}

None

\section{References}

[1] E. Monfrini, V. Melzi, G. Buongarzone, G. Franco, D. Ronchi, R. Dilena, E. Scola, P. Vizziello, A. Bordoni, N. Bresolin, G.P. Comi, S. Corti, A. Di Fonzo, A de novo C19orf12 heterozygous mutation in a patient with MPAN, Parkinsonism Relat Disord 48 (2018) 109-111.

[2] M.B. Hartig, A. Iuso, T. Haack, T. Kmiec, E. Jurkiewicz, K. Heim, S. Roeber, V. Tarabin, S. Dusi, M. Krajewska-Walasek, S. Jozwiak, M. Hempel, J. Winkelmann, M. Elstner, K. Oexle, T. Klopstock, W. Mueller-Felber, T. Gasser, C. Trenkwalder, V. Tiranti, H. Kretzschmar, G. Schmitz, T.M. Strom, T. Meitinger, H. Prokisch, Absence of an orphan mitochondrial protein, c19orf12, causes a distinct clinical subtype of neurodegeneration with brain iron accumulation, Am J Hum Genet 89(4) (2011) 543-50.

[3] A. Iuso, O.C. Sibon, M. Gorza, K. Heim, C. Organisti, T. Meitinger, H. Prokisch, Impairment of Drosophila orthologs of the human orphan protein C19orf12 induces bang sensitivity and neurodegeneration, PLoS One 9(2) (2014) e89439. 
[4] G. Landoure, P.P. Zhu, C.M. Lourenco, J.O. Johnson, C. Toro, K.V. Bricceno, C. Rinaldi, K.G. Meilleur, M. Sangare, O. Diallo, T.M. Pierson, H. Ishiura, S. Tsuji, N. Hein, J.K. Fink, M. Stoll, G. Nicholson, M.A. Gonzalez, F. Speziani, A. Durr, G. Stevanin, L.G. Biesecker, J. Accardi, D.M. Landis, W.A. Gahl, B.J. Traynor, W. Marques, Jr., S. Zuchner, C. Blackstone, K.H. Fischbeck, B.G. Burnett, Hereditary spastic paraplegia type 43 (SPG43) is caused by mutation in C19orf12, Hum Mutat 34(10) (2013) 1357-60.

[5] M. Gagliardi, G. Annesi, G. Lesca, E. Broussolle, G. Iannello, V. Vaiti, A. Gambardella, A. Quattrone, C19orf12 gene mutations in patients with neurodegeneration with brain iron accumulation, Parkinsonism Relat Disord 21(7) (2015) 813-6.

[6] P. Hogarth, A. Gregory, M.C. Kruer, L. Sanford, W. Wagoner, M.R. Natowicz, R.T. Egel, S.H. Subramony, J.G. Goldman, E. Berry-Kravis, N.C. Foulds, S.R. Hammans, I. Desguerre, D. Rodriguez, C. Wilson, A. Diedrich, S. Green, H. Tran, L. Reese, R.L. Woltjer, S.J. Hayflick, New NBIA subtype: genetic, clinical, pathologic, and radiographic features of MPAN, Neurology 80(3) (2013) 268-75.

[7] M.A. Dezfouli, A. Alavi, M. Rohani, M. Rezvani, T. Nekuie, B. Klotzle, S.H. Tonekaboni, G.A. Shahidi, E. Elahi, PANK2 and C19orf12 mutations are common causes of neurodegeneration with brain iron accumulation, Mov Disord 28(2) (2013) 228-32. 
A

\section{RDHT08}
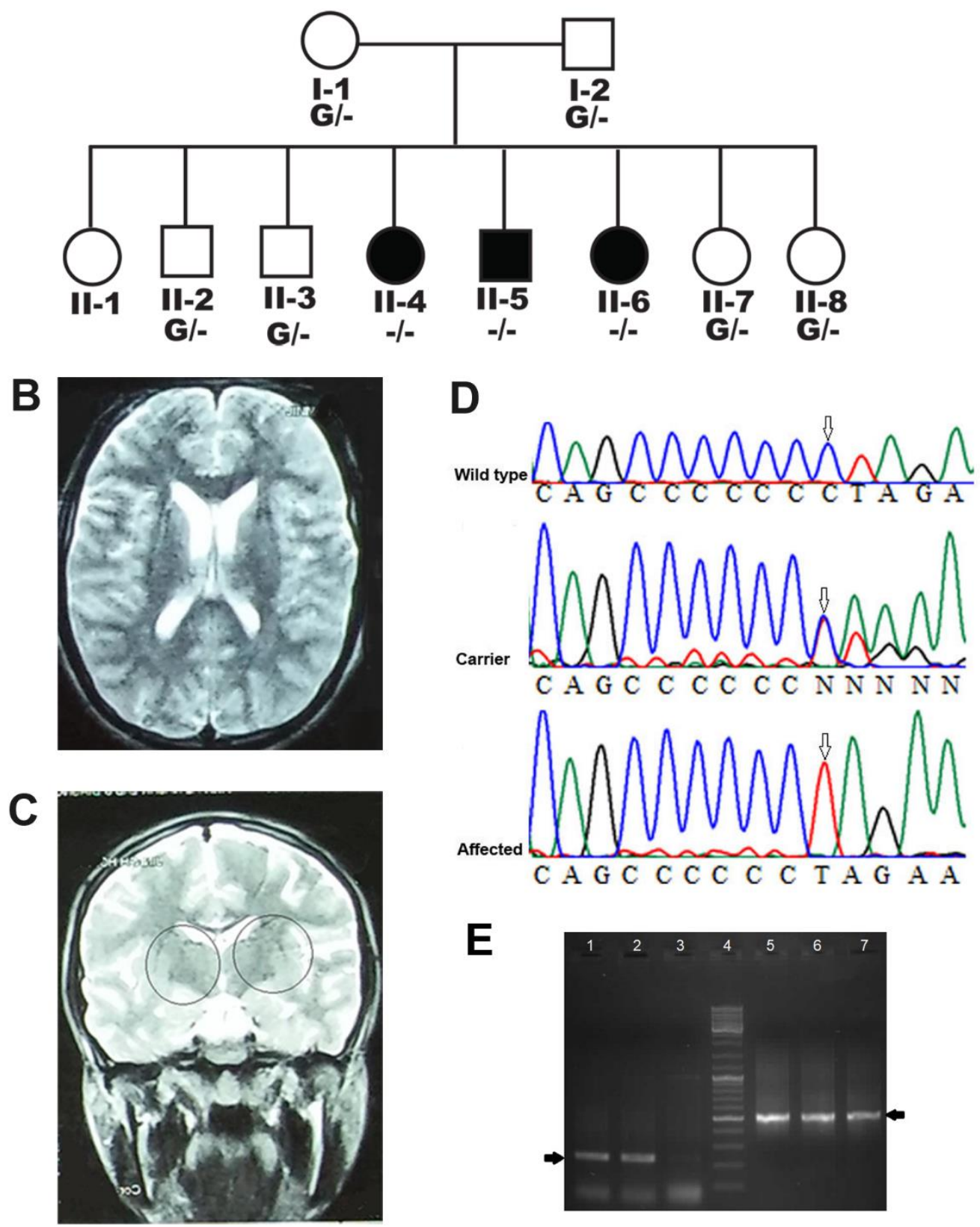

F

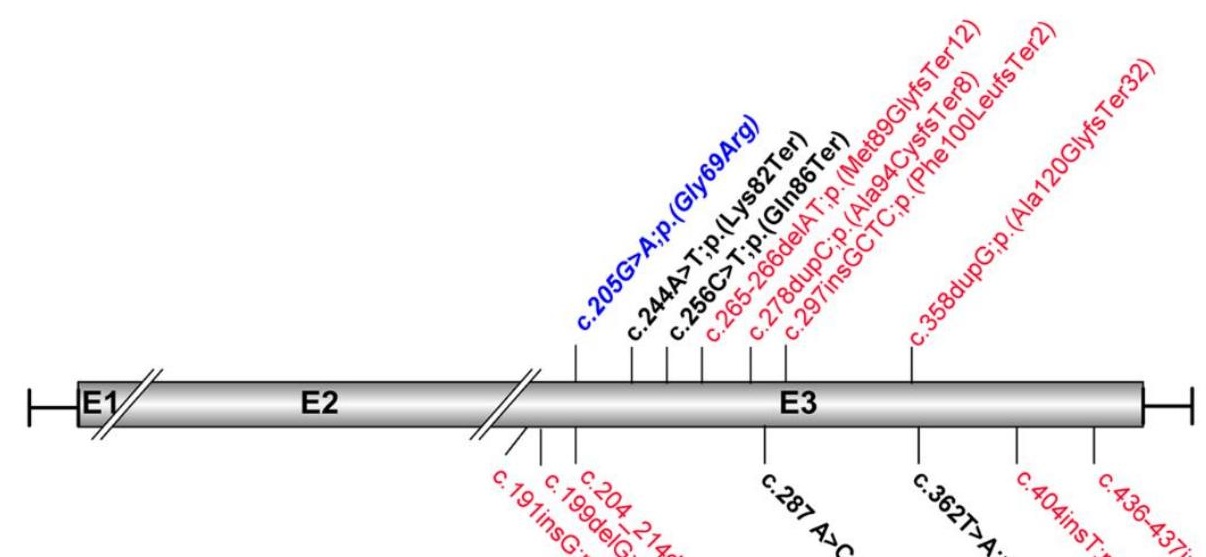

